\title{
Estudio de factores de riesgos ergonómicos presentes en la educación a distancia
}

\section{Study of ergonomic risk factors present in distance education}

\author{
Emili Araúz ${ }^{1}$, Carla Mojica ${ }^{1}$, Leonardo Zurdo ${ }^{1}$,Evidelia Gómez ${ }^{1 *}$ \\ ${ }^{I}$ Facultad de Ingeniería Industrial, Universidad Tecnológica de Panamá
}

\begin{abstract}
Resumen En esta investigación se presenta un estudio sobre la identificación de riesgos ergonómicos que están presentes en ambientes de trabajo y estudio en la educación a distancia que se implementó a causa del virus SARS-COV-2 el cual originó la pandemia de COVID-19. En Panamá a inicios del mes de marzo del año 2020, las autoridades universitarias desarrollaron un plan de contingencia para facilitar las clases a distancia a nivel nacional. Tanto docentes como estudiantes, crearon espacios de trabajo en sus residencias. Para el desarrollo de este estudio, se levantó un marco de referencia de docentes y estudiantes de la Universidad Tecnológica de Panamá, Centro Regional de Chiriquí, utilizando una encuesta que permitiera conocer e identificar aquellos factores de riesgos ergonómico-presentes. Los resultados muestran que los riesgos posturales y las condiciones ambientales (ruido, iluminación, temperatura) se encuentran presentes en los entornos virtuales de educación a distancia, debido principalmente a los siguientes factores de riesgo: espacio y mobiliario de trabajo inadecuado, periodos prolongados en la misma postura, iluminación incorrecta o insuficiente, ruido ambiental, exposición prolongada a pantallas de visualización de datos, generando afectaciones a la salud tanto de docentes como estudiantes, especialmente en cuello, hombros, espalda dorsal y lumbar.
\end{abstract}

Palabras clave Educación a distancia, ergonomía, factores de riesgo.

\begin{abstract}
This research presents a study on the identification of ergonomic risks that are present in work and study environments in distance education, due to the SARS-COV-2 virus which caused the COVID-19 pandemic. In Panama at the beginning of March 2020 , the university authorities developed a contingency plan to facilitate distance classes nationwide. Both, teachers and students tried to create workspaces in their residences. For the development of this study, a framework of teachers and students of the Technological University of Panama, Chiriquí Regional Center, was prepared using a survey that would allow knowing and identifying those present ergonomic risk factors. The results show that postural risks and environmental conditions (noise, lighting, temperature) are present in virtual distance education environments, mainly due to the following risk factors: inadequate workspace and furniture, prolonged periods in the classroom in the same posture, incorrect or insufficient lighting, ambient noise, prolonged exposure to data display screens, causing health effects for both teachers and students, especially neck, shoulders, back and lumbar spine.
\end{abstract}

Keywords Distance education, ergonomics, risk factors.

*Corresponding author: evidelia.gomez@utp.ac.pa

\section{Introducción}

En marzo del 2020 debido a la cuarentena total a causa de una pandemia provocada por el virus SARS-COV-2 que causa la enfermedad del COVID-19; se tomó la decisión de que las clases de nivel universitario se dieran a distancia, utilizando diversas plataformas digitales y el internet. Es así como docentes y estudiantes a nivel nacional, crearon sus espacios de trabajo para el proceso de enseñanza-aprendizaje.

Para el segundo semestre del año 2020 la Universidad Autónoma de Chiriquí (UNACHI) contaba con 13,325 estudiantes matriculados [1] y la Universidad de Panamá (UP) con 69,607 estudiantes matriculados [2]. Las Universidades públicas tienen la mayor cantidad de estudiantes universitarios del país, en la cual se incluye también la Universidad Tecnológica de Panamá (UTP). La población universitaria de la UTP representa aproximadamente el $18 \%$ de la población total universitaria, reportando una cifra de 23,229 estudiantes matriculados en el segundo semestre del 2020 a nivel nacional [3], contando así con 1,676 de docentes registrados a nivel nacional para el primer semestre del año 2019 que fue el último registro publicado [4].

Según el Consejo de la Asociación Internacional de Ergonomía (IEA), la ergonomía es: "una disciplina científica de carácter multidisciplinar, que estudia las relaciones entre el hombre, la actividad que realiza y los elementos del sistema en que se halla inmerso, con la finalidad de disminuir las cargas 
físicas, mentales y psíquicas del individuo y de adecuar los productos, sistemas, puestos de trabajo y entornos a las características, limitaciones y necesidades de sus usuarios; buscando optimizar su eficacia, seguridad, confort y el rendimiento global del sistema" [5],[7]. Los factores de riesgos ergonómicos son aquellas condiciones ambientales, organizacionales, y de contenido, que aumenta la probabilidad de causar daño en las personas [8].

Es importante analizar los factores relacionados con la postura tales como: el puesto de trabajo, los trastornos musculoesqueléticos y los riesgos asociados a las pantallas de visualización de datos (PVD). Dada la condición actual de las clases a distancia, es fundamental una buena postura ya que es beneficioso para la salud, además que con una postura correcta no aplicamos presión extra sobre los huesos y órganos, y así el cuerpo realiza el mínimo esfuerzo en esta modalidad educativa a distancia.

La prevención postural es de suma importancia para mantener hábitos que beneficien la salud corporal y no sólo en la comunidad educativa, sino la ciudadanía en general, tomando conciencia que mantener hábitos de posturas adecuadas es importante para la salud por ello se deben realizar descansos a lo largo del día.

De lo contrario provocarían problemas relacionados con los trastornos musculoesqueléticos que se pueden intensificar con el tiempo. Vivimos utilizando tanto la computadora como otras tecnologías muchas horas al día, el sobre uso y el sedentarismo conlleva un problema preocupante que son las patologías de postura corporal que se dan desde una edad temprana.

Esta razón es la que conlleva nuestro objetivo en este estudio para así identificar los factores de riesgos ergonómicos en docentes y estudiantes de la Universidad Tecnológica de Panamá, Centro Regional de Chiriquí bajo la modalidad de educación a distancia, lo cual implica corroborar cuales son los trastornos musculoesqueléticos más frecuentes en la modalidad de estudio a distancia, y cuáles son los hábitos que provocan dichos trastornos, además de investigar los efectos que causa estar mucho tiempo frente a una pantalla de visualización de datos (PVD), por ende, en este caso se estudian tres áreas de interés de la ergonomía que son:

a. Ergonomía ambiental,

b. Ergonomía temporal,

c. Ergonomía física del trabajo.

\subsection{Ergonomía ambiental}

La ergonomía ambiental es el área de la ergonomía que se encarga del estudio de las condiciones físicas que rodean al ser humano y que influyen en su desempeño al realizar las diversas actividades [9].

Algunas características que encontramos en el puesto de trabajo o en un área de estudio ha afectado la salud de muchas personas, por ende, están asociadas a lesiones la cual se les llama factores de riesgo de trabajo e incluye características físicas de la tarea.

Esta rama de la ergonomía se centraliza en el análisis del ambiente térmico, sonoro, vibratorio y lumínico donde la aplicación de los conocimientos de esta ayuda al diseño y evaluación de puestos y estaciones de trabajo, con el fin de incrementar el desempeño, seguridad y confort de quienes laboran en ellos [9].

\subsubsection{Ambiente lumínico}

La iluminación es una de las características más importante a la hora de tratar de ergonomía, ya que, influye en el ambiente de trabajo, sin embargo, al tener una iluminación inadecuada puede interferir en la adecuada visualización de los objetos y entornos, la eficiencia y eficacia del trabajador, en proporcionar la información adecuada y oportuna de señalización, además de que puede influir en el confort y salud visual en áreas de trabajos o estudio, provoca que esto afecte la salud de los trabajadores y el desarrollo del trabajo o tareas asignadas a causa de no cumplir con la iluminación adecuada [9].

El ambiente luminoso adecuado es uno de los agentes que más influyen en el confort de los puestos de trabajo y así mismo, en la reducción de los signos de fatiga. El trabajo con pantallas de visualización de datos tiene características específicas como el nivel de atención requerido y el tiempo de exposición frente a los monitores [10].

Por estas razones es necesario, realizar un espacio o ambiente de trabajo que tenga una iluminación óptima en los puestos de trabajo, para favorecer la percepción visual y asegurar una correcta ejecución de las tareas, la seguridad y bienestar de los trabajadores.

\subsubsection{Ambiente térmico}

Los estudios que se han realizado en esta área de la ergonomía ha contemplado el riesgo que representa en la salud y la incomodidad asociada con el trabajo en diversas y extremas condiciones climáticas, así como el cambio intermitente de temperaturas ambientales [9].

Como el ser humano tiene una temperatura inicial y el ambiente otra temperatura, se da un intercambio de temperatura entre el ambiente y la persona por ende en la ergonomía ambiental concretamente el ambiente térmico busca encontrar las condiciones térmicas adecuadas al momento de realizar su jornada de laboral.

\subsubsection{Ambiente sonoro}

El ruido se puede caracterizar psicológicamente por resultar molesto e indeseable, físicamente por su aleatoriedad espectral y de intensidades, y desde el punto de vista de la comunicación por su bajo o nulo contenido informativo. El 
tema de la relación entre el nivel de ruido, el tiempo de exposición y los daños físicos que causan al sistema auditivo en el humano ha sido estudiado con gran interés desde hace tiempo [9].

La función de los ergonomistas que se enfocan a esta área es la de encontrar la forma de reducir, aislar o controlar la emisión de ruido para lograr una condición ambiental óptima para el desempeño, salud y seguridad [9].

\subsubsection{Ambiente vibratorio}

El avance de la tecnología y su intervención en los puestos de trabajo ha provocado que más trabajadores estén expuestos a vibraciones, las cuales en algunos casos no tienen consecuencias, pero en otros puede afectar a la salud y capacidad de trabajo de quien se expone a ellas. Los pies, la zona de los glúteos y las manos, son las áreas del cuerpo que generalmente reciben $\mathrm{y}$ transmiten las vibraciones, dependiendo de la actividad que se realice y la posición en que se encuentre el operador, sin embargo, la exposición prolongada puede causar efectos crónicos que tienden a manifestarse después de un tiempo [9].

\subsection{Ergonomía temporal}

Muchos autores han mencionado diversas definiciones sobre que es ergonomía temporal, que se encarga del estudio de la relación de operador con los aspectos relativos al tiempo de trabajo [11].

Así como también es aquella que estudia la relación entre la fatiga y el descanso. Estudia la distribución semanal de la jornada laboral para evitar la fatiga física y mental, los tipos especiales de jornadas laborales, las vacaciones y los descansos [12].

Pero en resumen la ergonomía temporal es una simple relación de término, las cuales son el trabajo, estudio etc. Con el tiempo que se dedica para dicha acción; es decir, es un estudio basado en la cantidad de tiempo que se tiene en alguna actividad y como este se desarrolla ya sea de media o completa jornada, su solución básica radica en cómo repartir el tiempo en dichas jornadas para que no fuesen arduas para el operario y el trabajador, esto incluye también la distribución de tiempo como: horario de trabajo, ritmo de trabajo, descansos semanales y sobre todo vacaciones, sin embargo esto radica en cada cuanto debería seguir un tiempo de relajación implica las pausas que deberá tomar antes, durante y después del trabajo.

\subsubsection{Pausas y frecuencias de descansos}

Según la norma "ISO 11226:2000 - Ergonomics. Evaluation of static working postures" la cual menciona que como criterio técnico Se recomiendan pausas de unos 5 minutos o cambios posturales cada hora. Cuando la postura deba mantenerse de forma seguida, realizar micro pausas (unos segundos) cada 10 minutos. En ningún caso, debería prolongarse una misma posición de trabajo más de dos horas [13].

\subsection{Ergonomía física del trabajo}

Esta ergonomía es considerada como una de las más completas basándose en la comodidad del individuo en el trabajo, ya que valora el material de trabajo, la postura corporal durante el horario de trabajo, y el objetivo que tiene es el de evitar que puedan aparecer problemas físicos o bien mejorar el desarrollo del trabajo haciendo más funcional el puesto donde se labora y así prevenir los riesgos laborales.

Dentro de la ergonomía física se pueden distinguir los siguientes aspectos: la ergonomía operacional, ergonomía de seguridad y la ergonomía posicional.

Los puestos de trabajo se deben adaptar a cada individuo, puesto que trabajar en una postura incomoda no es una forma eficiente de trabajar. El proceso de ergonomía fomenta el trabajo en la "zona de confort", lo que causa menos fatiga y le ayuda a trabajar más rápido y con mayor precisión.

Cualquier parte del cuerpo es susceptible de padecer efectos nocivos, pero las más frecuentes son en el cuello, espalda, brazos y manos, y las causas varían. Las condiciones de trabajo son las que originan un gran número de ellos principalmente por posturas estáticas prolongadas, posturas incorrectas a causa de diseños incorrectos del puesto, movimientos repetitivos y levantamiento manual de cargas de forma incorrecta o con peso excesivo.

\subsubsection{Consecuencia de la postura inadecuada}

Los dolores musculoesqueléticos no solo ocurren en la tercera edad, sino en cualquier momento de la vida. Entre una de cada tres y una de cada cinco personas, incluidos los niños, sufren una afección osteoarticular o muscular dolorosa y discapacitante. Estos trastornos limitan enormemente la movilidad y la destreza, obligan a jubilaciones anticipadas, reducen la riqueza acumulada y afectan a la capacidad de participación en la vida social [14].

Las posibles consecuencias de una mala postura son el dolor lumbar, que suele darse cuando la persona permanece mucho tiempo sentada, con la espalda encorvada.

Las molestias en la zona central de la espalda, estas molestias son comunes cuando se sienta con una curva dorsal exagerada, con los hombros muy hacia el frente o, al estar de pie sacando barriga o por tiempo prolongado en esta posición. El dolor en cuello y parte superior de los hombros ocurre cuando hay un apoyo excesivo sobre las articulaciones de las últimas vértebras cervicales, por lo general cuando la persona se queda mirando al frente por tiempo prolongado [15]. 


\subsection{Investigaciones sobre modalidad presencial}

El desarrollo de las investigaciones sobre los riesgos ergonómicos en el sector educativo ha tomado un impulso notable en este año 2020, por lo cual muchos artículos se basan en datos ya establecidos en modalidad presencial.

En los últimos años se ha incrementado las alteraciones de la salud en los docentes en primaria durante el ejercicio de sus funciones los educadores están expuestos a múltiples problemas que van a afectar su salud, algunos relacionados con el estrés, este a su vez causa problemas fisiológicos derivados que son: dolores de cabeza frecuentes, mandíbula rígida, músculos tensos y contracturas musculares [16].

Por otro lado, estudios antropométricos relacionado con la educación a nivel secundario mencionan que debe haber una relación entre el mobiliario con los docentes y estudiantes [17].

Apoyándonos en los estudios de los riesgos ergonómicos en el sector educativo, en el cual menciona que los riesgos existentes se pueden clasificar de dos maneras que son: trastorno musculoesquelético y los riesgos asociados a las pantallas de visualización de datos [18] y a los trastornos musculoesqueléticos que son causados principalmente por hábitos posturales.

\section{Materiales y métodos}

La Universidad Tecnológica de Panamá sede regional de Chiriquí cuenta con una cantidad de 2,419 estudiantes matriculados y 185 docentes registrados según la página oficial de la UTP [3]. Esta investigación es exploratoria, pues para ello se utiliza la estadística descriptiva con un enfoque mixto tanto cualitativo como cuantitativo, ya que, se recopilan datos mediante una encuesta previamente diseñada.

Para escoger el tamaño de la muestra se utiliza la ecuación (1) obtenida en Survey Monkey [19] y la ecuación (2), es la que se utiliza para conocer si existe una relación entre las variables por medio del coeficiente de correlación que es: una medida de regresión que pretende cuantificar el grado de variación conjunta entre dos variables. Por tanto, es una medida estadística que cuantifica la dependencia lineal entre dos variables, es decir, si se representan en un diagrama de dispersión los valores que toman dos variables, el coeficiente de correlación lineal señalará lo bien o lo mal que el conjunto de puntos representados se aproxima a una recta, dicho de otra forma, es una manera de encontrar que tan relacionadas están las variables que se estén estudiando pero este dato se proporciona mediante el analizador de datos de Excel.

$$
\text { Tamaño de la muestra }=\frac{\frac{z^{2 *} p^{*}(1-p)}{e^{2}}}{1+\left(\frac{z^{2 * p(1-p)}}{e^{2 * N}}\right)}
$$

Siendo:

$\mathrm{N}$ el tamaño total de la población e el margen de error en porcentaje z desviación estándar

En este caso se utiliza un $95 \%$ de nivel de confianza lo cual equivale a una $\mathrm{z}$ de 1.96 .

Utilizando los datos de $n=2,419$ para estudiantes y $n=185$ para docentes, lo demás como constantes, considerando un margen de error del $5 \%$.

Reemplazando estos datos obtenemos que:

Para docentes

Tamaño de la muestra $=126(1)$

Para estudiantes

Tamaño de la muestra $=332(1)$

Los diagramas de dispersión posibles para diferentes valores de $\mathrm{r}$. El valor de $\mathrm{r}$ es la raíz cuadrada de $\mathrm{r} 2$. Es negativo si la pendiente es negativa y es positivo si la pendiente es positiva [20]. Entonces:

$$
\text { Coeficiente de correlación, } r= \pm \sqrt{r^{2}}
$$

Conociendo que el coeficiente de correlación va en un intervalo cerrado desde -1 hasta 1 , la manera correcta de interpretarlo es la siguiente:

1) $\mathrm{Si}$ el coeficiente de correlación en este caso denominado con la letra $\mathrm{r}$ es igual a 1 , se dice que la correlación es directa.

2) Si r está en un intervalo de 0 y 1 entonces, es una correlación positiva, esto quiere decir que las variables tienden a incrementarse de manera similar, con sus valores respectivos, además de que entre más se aproxime a 1 el valor, más fuerte es la relación entre las variables.

3) Si r es igual a 0 entonces no existe una relación, pero esto no implica que sean variables independientes.

4) Si r está en un intervalo de - 1 a 0 entonces, se interpreta que entre más se aproxima a 0 el valor, más débil es la relación entre las variables [21].

Sin embargo, dadas las circunstancias de la modalidad y las condiciones que se encontraba la población de muestra por la pandemia, el difícil acceso a la cantidad exacta de estudiantes y docentes, además del limitado tiempo para la recopilación de los resultados y sobre todo las formas de implementación de encuestas vía online ya que no todos cuentan con un servicio de internet estable, ya sea por la lejanía $\mathrm{u}$ otros factores que impiden su conexión a la web, complicaron la obtención de la cantidad de muestra proporcionada mediante la fórmula, por esto se ha optado por utilizar el método estadístico conocido como muestreo selección intencionada o mejor conocido como muestreo por conveniencia siendo este 
el que consiste en la elección por métodos no aleatorios de una muestra cuyas características sean similares a las de la población objetivo. En este tipo de muestreos la "representatividad" la determina el investigador de modo subjetivo, siendo este el mayor inconveniente del método ya que no podemos cuantificar la representatividad de la muestra [22]. Siendo la cantidad final recopilada de 20 docentes y 60 estudiantes los que respondieron la encuesta para la investigación.

No obstante, también se recomienda para efecto de realizar una investigación similar el método de muestreo aleatorio simple, cuya definición es: una manera de obtener una muestra representativa mediante el cual cada uno de los miembros de la población tiene la misma posibilidad de ser incluido en la muestra [23].

Para el procesamiento de los datos se utiliza el software llamado Untitled Form- Google Forms para realizar las encuestas vía online, el cual recopila datos y realiza gráficas de los resultados obtenidos, medidos en porcentajes y para el análisis de estos se utiliza el analizador de datos de Excel, en este caso se requirió para encontrar el coeficiente de correlación de algunas de las variables que se va a estudiar y además encontrar el tamaño adecuado de la muestra para el estudio.

\section{Resultados y discusión}

Se logra recompilar resultados de 80 personas divididas en 20 docentes y 60 estudiantes de la Universidad Tecnológica de Panamá, Centro Regional de Chiriquí con modalidad de educación a distancia cuyo único requisito para hacer la encuesta era la de ser estudiante o docente de la UTP sede regional de Chiriquí, independientemente a la facultad que perteneciera.

En la tabla 1 se puede observar las dolencias relacionadas con los trastornos musculoesqueléticos más frecuentes, hábitos ergonómicos practicados que han estado vigente en esta modalidad de educación a distancia y riesgos relacionados a pantallas de visualización de datos (PVD) de los docentes y estudiantes.
Tabla 1. Factores más frecuentes en la modalidad de estudios a distancia

\begin{tabular}{|c|c|c|c|}
\hline \multicolumn{2}{|r|}{ Factor } & $\begin{array}{c}\text { Docentes } \\
(\%)\end{array}$ & $\begin{array}{c}\text { Estudiantes } \\
(\%)\end{array}$ \\
\hline \multirow{7}{*}{ 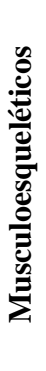 } & $\begin{array}{l}\text { Cuello, hombros y/o } \\
\text { espalda dorsal }\end{array}$ & 80 & 68.3 \\
\hline & Espalda lumbar & 65 & 60 \\
\hline & Codos & 25 & 13.3 \\
\hline & Manos y/o muñecas & 40 & 11.7 \\
\hline & Piernas & 30 & 23.3 \\
\hline & Rodilla & 20 & 20 \\
\hline & Pies & 10 & 10 \\
\hline \multirow{9}{*}{$\frac{\mathscr{n}}{\frac{\pi}{20}}$} & $\begin{array}{l}\text { Mantenimiento } \\
\text { excesivo de una } \\
\text { posición erguida }\end{array}$ & 40 & 26.7 \\
\hline & $\begin{array}{l}\text { Mantenimiento } \\
\text { excesivo de una } \\
\text { posición sentada }\end{array}$ & 65 & 70 \\
\hline & $\begin{array}{c}\text { Flexión inadecuada de } \\
\text { la columna }\end{array}$ & 40 & 53.3 \\
\hline & $\begin{array}{c}\text { La adopción de mala } \\
\text { postura al sentarse }\end{array}$ & 50 & 63.3 \\
\hline & $\begin{array}{l}\text { El mantenimiento de } \\
\text { una postura sentada } \\
\text { estática prolongada }\end{array}$ & 65 & 33.3 \\
\hline & $\begin{array}{l}\text { Los movimientos } \\
\text { repetitivos debidos al } \\
\text { manejo habitual e } \\
\text { intensivo del teclado y } \\
\text { el ratón }\end{array}$ & 60 & 38.3 \\
\hline & $\begin{array}{l}\text { Excesiva flexión o } \\
\text { torsión del cuello al } \\
\text { escribir o mirar la } \\
\text { pantalla, } \\
\text { respectivamente }\end{array}$ & 45 & 43.3 \\
\hline & $\begin{array}{l}\text { Falta de apoyo de } \\
\text { brazos y muñecas } \\
\text { mientras se teclea }\end{array}$ & 45 & 35 \\
\hline & $\begin{array}{c}\text { Falta de sitio para } \\
\text { apoyar las muñecas y } \\
\text { desviación cubital de } \\
\text { las manos al teclear }\end{array}$ & 30 & 30 \\
\hline \multirow{5}{*}{ 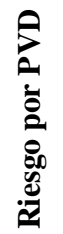 } & Ojos llorosos & 65 & 58.3 \\
\hline & Ojos secos & 60 & 31.7 \\
\hline & Visión borrosa & 65 & 60 \\
\hline & Doble visión & 20 & 31.7 \\
\hline & Ardor & 50 & 56.7 \\
\hline
\end{tabular}

Como se muestra las dolencias que inciden en los docentes y estudiantes son: cuello, hombros y/o espalda dorsal y la espalda lumbar esto es debido a ciertos hábitos practicados, 
tales como tener un mantenimiento excesivo de una posición sentada la cual provoca las molestias mencionadas.

Otro mal hábito en los docentes es el mantenimiento de una postura estática prolongada y los movimientos repetitivos debido al manejo habitual e intensivo del teclado y el ratón, provocándoles dolores en las manos y/o muñecas.

De igual forma, la flexión inadecuada de la columna y la adopción de una mala postura al sentarse causa a los estudiantes dolencias en las piernas y rodillas.

Estas dolencias mencionadas en la tabla 1 son debido a dos factores, la primera es la que se menciona en la figura 1 donde podemos observar la comparación de horas dedicadas a clases por día, de estudiantes y docentes lo que dio como resultados que el $20 \%$ de los docentes y $37 \%$ de los estudiantes de la muestra dedican entre 4 a 6 horas al día.

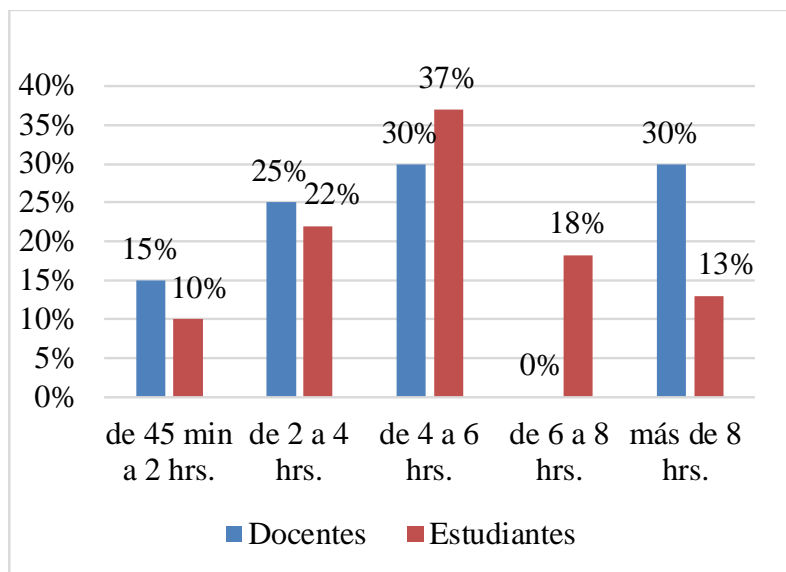

Figura 1. Porcentaje de los docentes y estudiantes según la cantidad de horas dedicadas a clases por día.

El segundo factor es debido al tiempo de intervalo de descanso durante su jornada de clases, estudios, etc. Mostrados en la figura 2 lo que da como resultado que el $52.6 \%$ de los docentes optan por hacer descansos de menos de 10 minutos y en los estudiantes un $37 \%$ entre 10 a 15 minutos.

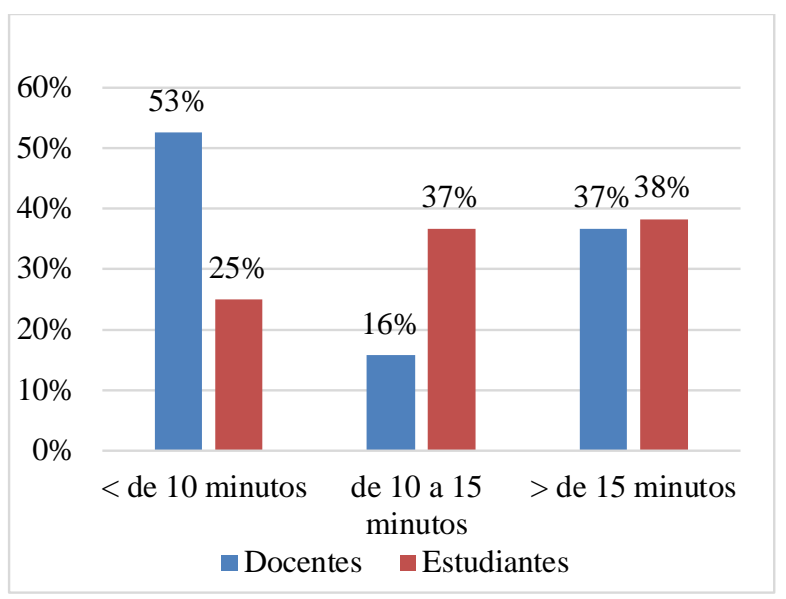

Figura 2. Porcentaje de los docentes y estudiantes según el tiempo de descanso.

Adicional a la información de las gráficas anteriores, en la figura 3 se destaca que tanto el $51.7 \%$ de los docentes y el $30 \%$ de los estudiantes pasan más de 8 horas frente a una PVD, lo cual provoca las molestias presentadas en la figura 4, en donde los ojos llorosos y visión borrosa fueron los valores más altos después de haber culminado sus actividades diarias.

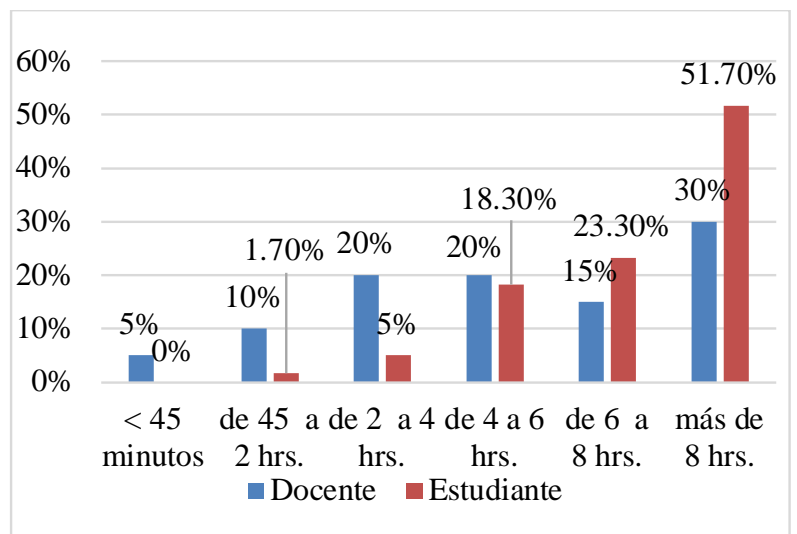

Figura 3. Porcentaje de docentes y estudiantes según el tiempo de exposición frente a las PVD. 


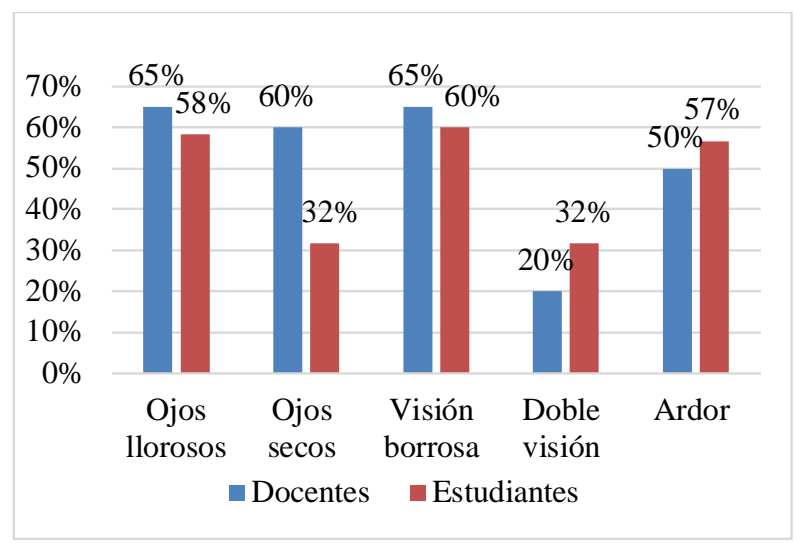

Figura 4. Porcentaje de docentes y estudiantes según las molestias experimentadas por la exposición a las PVD.

Tabla 2. Observaciones del ambiente de trabajo y las PVD

\begin{tabular}{|c|c|c|c|}
\hline \multicolumn{4}{|c|}{ Resultados sobre las observaciones en: } \\
\hline & & $\begin{array}{c}\text { Docentes } \\
(\%)\end{array}$ & $\begin{array}{c}\text { Estudiantes } \\
(\%)\end{array}$ \\
\hline \multirow{8}{*}{ 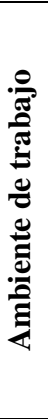 } & $\begin{array}{l}\text { Tipo de mesa: } \\
\text { Escritorio }\end{array}$ & 85 & 45 \\
\hline & Tipo de mesa: Otros & 15 & 55 \\
\hline & Tipo de silla: Oficina & 60 & 33.3 \\
\hline & Tipo de silla: Otros & 40 & 66.7 \\
\hline & \multirow{2}{*}{$\begin{array}{c}\text { Libertad de } \\
\text { movimiento y postura } \\
\text { confortable }\end{array}$} & 78.9-Sí & 51.7-Sí \\
\hline & & 21.1-No & 48.3-No \\
\hline & \multirow{2}{*}{$\begin{array}{l}\text { Permite altura de } \\
\text { asiento ajustable }\end{array}$} & 60-Sí & 33.3-SÍ \\
\hline & & $40-\mathrm{No}$ & $66.7-\mathrm{No}$ \\
\hline \multirow{8}{*}{$\sum_{2}^{2}$} & \multirow{2}{*}{$\begin{array}{l}\text { Superficie de la mesa } \\
\text { permite reflejos }\end{array}$} & 25-Sí & 80-Sí \\
\hline & & $75-\mathrm{No}$ & $20-\mathrm{No}$ \\
\hline & \multirow{2}{*}{$\begin{array}{l}\text { Área de trabajo lo } \\
\text { suficientemente amplia }\end{array}$} & 65-Sí & 61.7-Sí \\
\hline & & $35-\mathrm{No}$ & 38.3-No \\
\hline & \multirow{2}{*}{ Iluminación artificial } & 65-Sí & 61-Sí \\
\hline & & $35-\mathrm{No}$ & 39-No \\
\hline & \multirow{2}{*}{ Iluminación directa } & 70-Sí & 45-Sí \\
\hline & & $30-\mathrm{No}$ & $55-\mathrm{No}$ \\
\hline
\end{tabular}

En la tabla 2 encontramos el tipo de ambiente de trabajo e información acerca de la pantalla de visualización de datos los cuales han sido optados por los docentes y estudiantes en la modalidad a distancia. En contraste con lo investigado sobre ergonomía en el sector educativo, cabe destacar una similitud con las clases presenciales pese a las diferencias de modalidad enseñanza-aprendizaje se sigue manteniendo constante la fatiga muscular ya que la gran mayoría de los encuestados presentaba dolor en las mismas zonas musculares debido a una ausencia de periodo de descanso, siendo este el causante de contracciones musculares continuas que impiden la adecuada oxigenación.

Dentro del estudio es posible hacer una relación entre las dolencias corporales con los hábitos ergonómicos de los docentes y estudiantes, ya que pese a corroborar que existe un porcentaje alto de docentes y estudiantes que cuentan con un ambiente laboral adecuado, esto no explica el porqué de las dolencias presentadas, pero al estudiar cuales podrían ser las razones, encontramos que por ejemplo: el dolor de espalda dorsal se debe a que hay una excesiva flexión o torsión del cuello al escribir o mirar la pantalla, respectivamente, con respecto a la espalda lumbar varia de dos formas las que son: mantenimiento excesivo de una posición erguida y sentada la segunda razón se debe a una flexión inadecuada de la columna, manos fácilmente radica en los movimientos repetitivos debidos al manejo habitual e intensivo del teclado y el ratón por otro lado los codos es por la falta de apoyo de brazos y muñecas mientras se teclea además de una desviación cubital de las manos al teclear.

Dichas relaciones son presentadas en las gráficas a continuación, las cuales contemplan:

a) Las dolencias visuales en contraste con el tiempo de exposición frente a un PVD en los docentes y estudiantes.

b) Las dolencias corporales con los hábitos ergonómicos de los docentes y estudiantes

Como se puede observar en las figuras 5 y 6 el coeficiente de determinación es de un $88 \%$ en docentes y $78 \%$ en estudiantes y para el coeficiente de correlación sacando la raíz de estos obtenemos que para docentes es de 0.94 y para estudiantes es de 0.89 conociendo que el $85 \%$ de los docentes encuestados tiene un rango de edad mayor a 40 y el $90 \%$ de los estudiantes están en un rango de edad de entre los 18 a 24 años, lo cual puede implicar que los docentes tengan está cifra más alta debido a la edad.

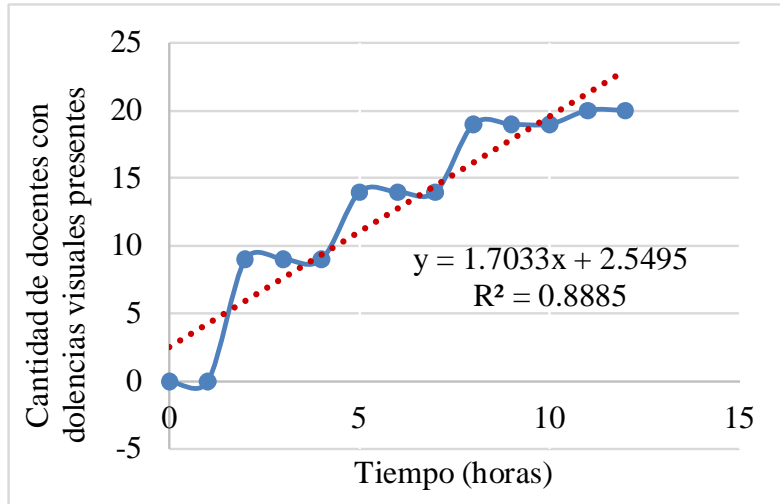

Figura 5. Cantidad de docentes con dolencias visuales según el tiempo de exposición frente a PVD. 


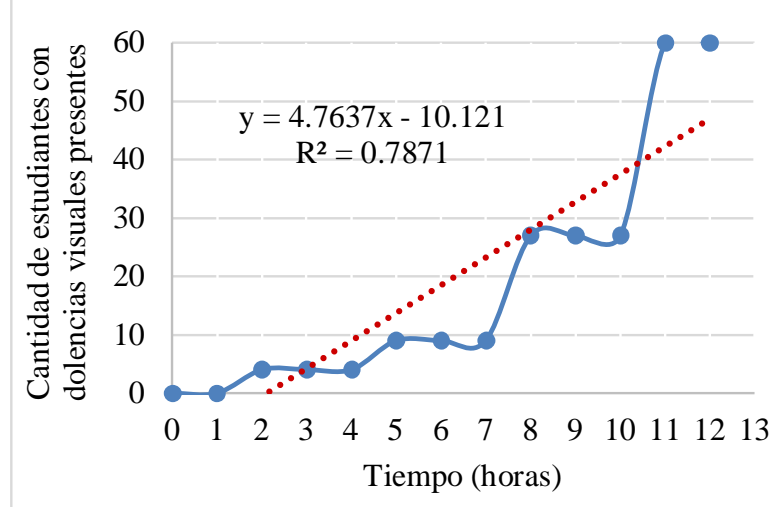

Figura 6. Cantidad de estudiantes con dolencias visuales según el tiempo de exposición frente a PVD.

En las figuras 7 y 8 se muestra cuáles son las consecuencias por mantener una posición determinada o movimientos repetitivos.

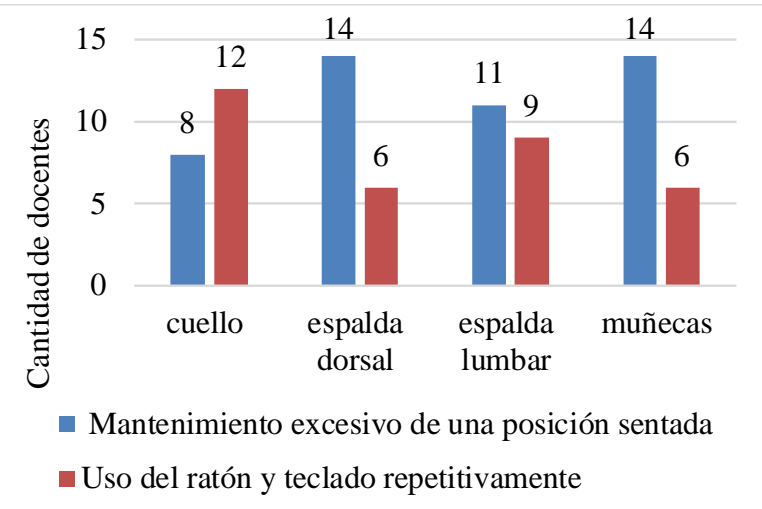

Figura 7. Hábitos practicados vs dolencias provocadas en docentes.

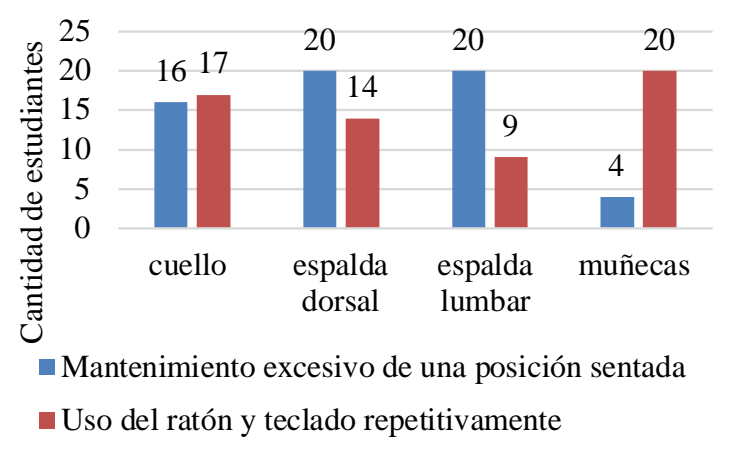

Figura 8. Hábitos practicados vs dolencias provocadas en estudiantes.
Además, como se puede observar que existe una relación directamente proporcional a los trastornos músculosesqueléticos ya mencionados con los malestares y/o dolencias visuales que produce el uso de las pantallas de visualización de datos (PVD) mostradas en la tabla 1, según un estudio de riesgos ergonómicos [24] el cual menciona que estos son provocados por el brillo de la pantalla ya sea por la cantidad de luz emitida, la distancia entre los ojos y la pantalla o documento, el tiempo de exposición a la fuente luminosa, la duración y frecuencia de pausas y descansos.

\section{Conclusiones}

En esta investigación se identificaron cuáles son los factores de riesgos ergonómicos presentes en la educación a distancia: problemas relacionados con trastornos musculoesqueléticos y con respecto a las pantallas de visualización de datos y cuáles de ella son las que se presentan en cifras más altas, también se registró cuáles eran los hábitos ergonómicos presentes en esta modalidad.

Por último, se conoció cual es el tipo de ambiente laboralestudio de los docentes y estudiantes, además que al este presentar una amplia variedad en resultados también podemos arraigar que, aunque exista un ambiente laboral adecuado el no cumplir con los descansos necesarios para el cuerpo y vista, se convertiría en el factor más influyente en el estudio.

Como aporte se puede mencionar que los resultados de este estudio pueden proporcionar información valiosa a la universidad, que sirven de base para brindar medidas de prevención de trabajo tanto para docentes como estudiantes. Igualmente, la metodología puede ser aplicada a actividades laborales presenciales y a distancia.

Asimismo, conocer los niveles de riesgos de estos factores y encontrar si existe una relación directa entre los dolores musculoesqueléticos con el rendimiento académico de los estudiantes.

\section{REFERENCIAS}

[1] Universidad Autónoma de Chiriquí, "Cuadro 187-01. Matrícula total por sexo, según sede, facultad y carrera: I semestre 2020”. 2020, [En línea]. Disponible en:

http://www.unachi.ac.pa/assets/descargas/planificacion/18701_2020.pdf.

[2] Universidad de Panamá, "Matrícula de pregrado y grado en la Universidad de Panamá, según sede, facultad y ubicación: primer semestre; año académico (P)”. 2020, [En línea]. Disponible en: https://www.up.ac.pa/sites/default/files/202008/MATRI. PRELI.PAGINA WEB-17-AGOSTO-2020.pdf.

[3] Universidad Tecnológica de Panamá, "Matrícula por sexo, según sede: segundo semestre 2020”. 2020, [En línea]. Disponible en: 
https://utp.ac.pa/sites/default/files/documentos/2021/pdf/utpmatricula-sexo-sede-2dosemestre2020-2021.pdf.

[4] Universidad Tecnológica de Panamá, "Personal docente por tiempo de dedicación y sexo, según sede: primer semestre 2019”. 2019, [En línea]. Disponible en:

https://utp.ac.pa/documentos/2020/pdf/docentes-dedicacion-sexosede-1er19.pdf.

[5] Consejo de Salud Ocupacional, "Conceptualización de la Ergonomía”, 2021. "[En 1 http://dianelos.com/temas_de_interes/ergonomia.aspx [Accedido mar. 24, 2021].

[6] Real Academia Española, "Ergonomía", Diccionario de la lengua española - Edición del Tricentenario. 2021, Accedido: mar. 24, 2021. [En línea]. Disponible en: https://dle.rae.es/ergonomía.

[7] International Ergonomics Association, "What Is Ergonomics?" https://iea.cc/what-is-ergonomics/ (accedido mar. 24, 2021).

[8] S. J. Cachay Nascimento, H. Heredia Arévalo, y D. V. Zegarra Papa, "Factores de riesgos ergonómicos y sintomatologías músculo - esqueléticas en enfermeras asistenciales del Hospital Regional de Loreto, Iquitos 2017”, Universidad Nacional de la Amazonía Peruana, 2017.

[9] EcuRed, "Ergonomía ambiental", 2019. https://www.ecured.cu/Ergonom\%C3\%ADa_ambiental (accedido mar. 24, 2021).

[10] A. P. Geraldo y G. M. Paniza, "Ergonomía ambiental: Iluminación y confort térmico en trabajadores de oficinas con pantalla de visualización de datos”, Revista Ingeniería, Matemáticas y Ciencias de la Información, vol. 1, n.o 2, Art. n.o 2, ago. 2014, Accedido: mar. 24, 2021. [En línea]. Disponible en: http://ojs.urepublicana.edu.co/index.php/ingenieria/article/vie $\mathrm{w} / 228$.

[11] D. G. Maestre, Ergonomía y psicosociología. FC Editorial, 2007.

[12] A. Méndez González, "Ergonomía cognitiva - crono ergonomía - ergonomía temporal turnos rotativos - análisis y soluciones de gestión”, Universidad Miguel Hernández, 2020.

[13] International Organization for Standardization, "ISO 11226:2000 (en), Ergonomics — Evaluation of static working postures",

2000.
[14] Organización Mundial de la Salud, "Trastornos musculoesqueléticos", 2021. https://www.who.int/es/newsroom/fact-sheets/detail/musculoskeletal-conditions (accedido mar. 24, 2021).

[15] Clínicas Premium Madrid, "Cómo afecta tu Postura a tu día a día”, Rehabilitación Premium Madrid, nov. 12, 2020. https://rehabilitacionpremiummadrid.com/blog/lauramartin/postura-consecuencias/ (accedido mar. 24, 2021).

[16] E. Escalona, "Relación salud-trabajo y desarrollo social: visión particular en los trabajadores de la educación", Revista Cubana de Salud Pública, vol. 32, n.o 1, pp. 0-0, mar. 2006.

[17] J. Rojas Colvin, A. A. Almagià Flores, y J. S. Ilardi, "Estudio Antropométrico en Párvulos Atendidos por el Sistema Educativo Público Chileno para el Diseño de Mobiliario", International Journal of Morphology, vol. 31, n.o 1, pp. 189196, mar. 2013, doi: 10.4067/S0717-95022013000100032.

[18] Secretaría de Salud Laboral y Medio Ambiente, "Por la mejora de las condiciones de seguridad y salud en el trabajo. Los Riesgos Ergonómicos en el sector Educativo". 2009, [En línea]. Disponible en: http://portal.ugt.org/saludlaboral/publicaciones_new/files_fete _riesgosergofete/publication.pdf.

[19] SurveyMonkey, "Calculadora del tamaño de muestra", SurveyMonkey, 2021. https://es.surveymonkey.com/mp/sample-size-calculator/ (accedido mar. 24, 2021).

[20] B. Render, R. M. Stair, y M. E. Hanna, Métodos cuantitativos para los negocios, 11.a ed. Pearson Educación, 2012.

[21] Economipedia, "Coeficiente de correlación lineal - Definición, qué es y concepto", Economipedia, dic. 17, 2015. https://economipedia.com/definiciones/coeficiente-decorrelacion-lineal.html (accedido mar. 24, 2021).

[22] Jordi Casal y Enric Mateu, "Tipos de muestreo", Rev. Epidem. Med. Prev., vol. 1, pp. 3-7, 2003.

[23] Murray R. Spiegel y Larry J. Stephens, Estadística, 4.a ed. McGraw-Hill, 2009.

[24] Fundación para la Prevención de Riesgos Laborales, "Pantallas Visualización de Datos (PVD) - Riesgos Laborales", 2015. https://riesgoslaborales.saludlaboral.org/portalpreventivo/riesgos-laborales/riesgos-relacionados-con-lahergonomia/pantallas-visualizacion-de-datos-pvd/ (accedido mar. 24, 2021) 